\title{
Acute effects of whole body vibration exercise on post-load glucose metabolism in healthy men: a pilot randomized crossover trial
}

\author{
Hirotaka Watanabe ${ }^{1} \cdot$ Mitsuyoshi Takahara $\mathbb{C}^{1,2} \cdot$ Naoto Katakami $^{1} \cdot$ Takashi Kanamoto $^{3} \cdot$ Ken Nakata $^{3} \cdot$ \\ lichiro Shimomura ${ }^{1}$
}

Received: 20 May 2021 / Accepted: 23 September 2021 / Published online: 2 October 2021

(c) The Author(s), under exclusive licence to Springer Science+Business Media, LLC, part of Springer Nature 2021

\begin{abstract}
Purpose Exercise on a whole body vibration (WBV) platform, namely WBV exercise (WBVE), has long-term beneficial effects on glucose metabolism, similarly to conventional moderate-intensity exercise. Conventional moderate-intensity exercise reduces post-load plasma glucose levels at the acute phase. This study aimed to reveal acute effects of WBVE on post-load glucose metabolism.

Methods This randomized crossover trial enrolled 18 healthy men. They completed the following three interventions in a random order: (1) a 2-hour 75-g oral glucose tolerance test (OGTT) without WBVE (OGTT-alone), (2) 20-minute WBVE before an OGTT (WBVE $\rightarrow$ OGTT), and (3) 20-minute WBVE during an OGTT (OGTT $\rightarrow$ WBVE). Post-load glucose metabolism in the WBVE $\rightarrow$ OGTT and OGTT $\rightarrow$ WBVE interventions were compared with that in the OGTT-alone intervention.

Results Plasma glucose levels in the WBVE $\rightarrow$ OGTT and OGTT $\rightarrow$ WBVE interventions were not significantly different from those in the OGTT-alone intervention at any time point except $15 \mathrm{~min}$, wherein the WBVE $\rightarrow$ OGTT intervention had higher glucose levels (111 [interquartile range, 102-122] mg/dL vs 122 [111-134] mg/dL, $P=0.026$ ). Higher plasma glucagon levels were observed at $0 \mathrm{~min}$ in the WBVE $\rightarrow$ OGTT intervention and at $60 \mathrm{~min}$ in the OGTT $\rightarrow$ WBVE intervention $(P=0.010$ and 0.015$)$. Cortisol, Growth hormone, and adrenaline levels were significantly increased after WBVE, whereas noradrenaline levels were not. Serum insulin levels in the WBVE $\rightarrow$ OGTT intervention were significantly higher than those in the OGTT-alone intervention at $0 \mathrm{~min}(P=0.008)$.

Conclusions WBVE did not decrease post-load plasma glucose levels at the acute phase. Acute effects of WBVE on post-load glucose metabolism would not be identical to those of conventional exercise.

The unique trial number and the name of the registry: UMIN000036520, www.umin.ac.jp, date of registration, June 12, 2019.
\end{abstract}

Keywords Whole body vibration exercise $\cdot$ Healthy men $\cdot$ Glucose metabolism $\cdot$ Acute phase

Supplementary information The online version contains supplementary material available at https://doi.org/10.1007/s12020021-02893-w.

Mitsuyoshi Takahara

takahara@endmet.med.osaka-u.ac.jp

1 Department of Metabolic Medicine, Osaka University Graduate School of Medicine, 2-2 Yamadaoka, Suita, Osaka 565-0871, Japan

2 Department of Diabetes Care Medicine, Osaka University Graduate School of Medicine, 2-2 Yamadaoka, Suita, Osaka 5650871, Japan

3 Department of Health and Sport Sciences, Osaka University Graduate School of Medicine, 2-2 Yamadaoka, Suita, Osaka 5650871, Japan

\section{Introduction}

Physical exercise is a key factor to reduce the risk of type 2 diabetes mellitus [1-3]. Exercise on a whole body vibration (WBV) platform, namely WBV exercise (WBVE), is a convenient, safe, and effective exercise modality, and has become popular in recent years. WBV enhances muscle activity, using mechanical loading of alternative gravitational acceleration with three-dimensional vibration $[4,5]$. WBV platforms for home use are now commercially available, and attract increasing attention during this coronavirus pandemic, when citizens are asked to stay at home and refrain from going out. Clinical studies demonstrated that WBVE had long-term beneficial effects, including 
Fig. 1 Study design

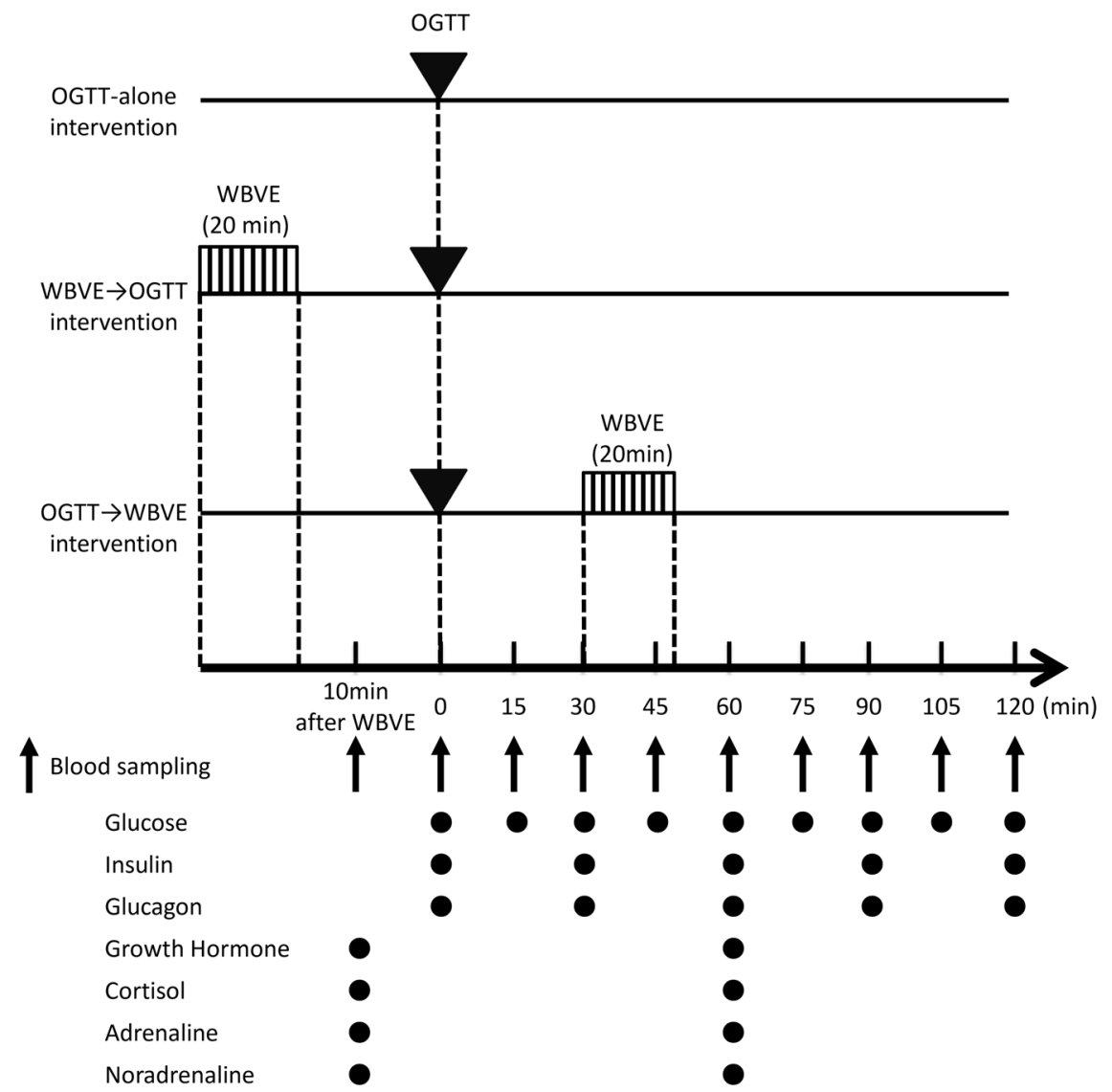

decrease of body fat mass [6], increase of muscle mass [7], and improvement of insulin sensitivity and glucose regulation [8], similarly to conventional moderate-intensity exercise [9].

However, it remained unrevealed how WBVE would affect glucose metabolism at the acute phase. The literature [10-14] shows that in comparison with rest, conventional moderate-intensity exercise, either following or followed by glucose ingestion, lowers post-load plasma glucose levels at the acute phase. By contrast, no data were so far available about the acute phase influence of WBVE on post-load glucose levels. The aim of the current pilot study was to investigate the acute effects of WBVE either following or followed by glucose ingestion on post-load glucose metabolism in healthy men, in comparison with rest as the control.

\section{Materials and methods}

\section{Study design}

This pilot study was an open-label, randomized, 6sequence, 3-period, 3-intervention crossover trial with a 1:1:1:1:1:1 allocation, and was conducted at Osaka
University, Suita City, Osaka, Japan, between July 2019 and July 2020. This study compared the acute effect on glucose metabolism between WBVE and rest in healthy men. Eighteen healthy male volunteers were enrolled. Each study participant received all of the following three interventions in a random order: (1) performing a 2-hour 75-g oral glucose tolerance test (OGTT) without any exercise including WBVE (OGTT-alone), (2) performing 20-minute WBVE before an OGTT (WBVE $\rightarrow$ OGTT), and (3) performing 20-minute WBVE during an OGTT (OGTT $\rightarrow$ WBVE) (Fig. 1). OGTTs were started at approximately 10:00 AM after an overnight fast. During an OGTT, blood samples were collected at $0 \mathrm{~min}$ and thereafter every $15 \mathrm{~min}$ for $2 \mathrm{~h}$. In the OGTT $\rightarrow$ WBVE intervention, WBVE was started $30 \mathrm{~min}$ after glucose ingestion. The timing was determined in reference to the optimal timing of post-meal conventional exercise [15]. By contrast, we found no general consensus about the optimal timing of pre-load or pre-meal exercise [15, 16]. We therefore assigned different WBVE-to-OGTT intervals to the study participants in the WBVE $\rightarrow$ OGTT intervention, to supplementarily analyze the association between the interval and study outcomes. The interval was ranged from 10 to $60 \mathrm{~min}$, which was randomly set in each participant. A blood sample was additionally collected $10 \mathrm{~min}$ after 
WBVE in the WBVE $\rightarrow$ OGTT intervention. The interval between each intervention was at least two days.

The study was in accordance with the Declaration of Helsinki, and approved by the Institutional Review Board of Osaka University Hospital (date of approval, May 10, 2019; approval number, 18452-3). All of the participants provided written informed consent prior to participation. The trial was registered as UMIN000036520 at UMIN Clinical Trials Registry.

\section{Study participants and allocation}

Eighteen healthy male volunteers aged 20-59 years were enrolled to the study. The exclusion criteria included the history of diabetes or glucose intolerance, diseases potentially related to abnormal glucose homeostasis, symptoms potentially deteriorated by vibration (dizziness etc.), and a risk of fall. The current cross-over trial had 6 sequences, each of which consisted of 3 interventions (OGTT-alone, $\mathrm{WBVE} \rightarrow \mathrm{OGTT}$, and OGTT $\rightarrow \mathrm{WBVE}$ ) in a different order. The study participants were allocated to one of the 6 sequences based on a 1:1:1:1:1:1-ratio randomization. The allocation concealment was ensured by using sequentially numbered opaque sealed envelopes (SNOSE). In brief, sealed opaque envelopes, each of which contained a card indicating one of the 6 sequences, were prepared and shuffled in advance of the clinical trial by one researcher (M.T.). Once a participant consented to participate in the clinical trial, a sealed opaque envelope was opened by another researcher (H.W.), and the participant was assigned to the sequence that was indicated by the card enclosed in the envelope. Sequences and interventions were opened (i.e., not blinded) after the assignment.

\section{WBVE}

WBVE was performed using a WBV platform (Personal Power Plate ${ }^{\varpi}$, Performance Health Systems, LLC, Northbrook, IL), which mechanically generated three dimensional (i.e., vertical, horizontal, and sagittal) WBV load. The duration and the type of WBVE were determined by reference to previous reports including Bellia et al. [8], which demonstrated long-term beneficial effects on insulin sensitivity and glucose regulation. In brief, the subjects performed a 60-second static squat on the platform 10 times with a 60-second rest between each squat. During a static squat, the subjects stood on the platform keeping their knees slightly bent (knee angle of $90-110^{\circ}$ flexion), with their feet shoulder-width apart. The pre-set frequency of the WBV platform was $35 \mathrm{~Hz}$. The vertical amplitude of WBV was 2-4 mm, whereas that of the other axes (horizontal and sagittal) was approximately one-seventh of the vertical amplitude.

\section{Measurement}

All blood samplings were performed from the indwelling venous catheter. The samples were immediately centrifuged at $1000 \mathrm{rpm}$ for $10 \mathrm{~min}$ at $4{ }^{\circ} \mathrm{C}$, and extracted plasma and serum were stored at $-20^{\circ} \mathrm{C}$. Plasma glucose levels were measured at $0 \mathrm{~min}$ and thereafter every $15 \mathrm{~min}$, and serum insulin and plasma glucagon levels were measured at $0 \mathrm{~min}$ and thereafter every 30 min during a 2-hour OGTT. Plasma glucose was measured using a hexokinase activity assay kit (Kanto Chemical Co., Inc., Tokyo, Japan), and serum insulin was measured using a chemiluminescent enzyme immunoassay kit (Fujirebio Inc., Tokyo, Japan). Glucagon levels were measured using a sandwich enzyme-linked immunosorbent assay kit (Mercodia AB, Uppsala, Sweden) [17, 18]. Plasma cortisol, growth hormone (GH), noradrenaline and adrenaline levels were measured $10 \mathrm{~min}$ after WBVE in the WBVE $\rightarrow$ OGTT and the OGTT $\rightarrow$ WBVE interventions, and at the corresponding time points in the OGTT-alone intervention (Fig. 1). Cortisol and growth hormone levels were measured using an electro chemiluminescence immunoassay kit (Roche Diagnostics K.K, Tokyo, Japan), and noradrenaline and adrenaline levels were measured using a high performance liquid chromatography method (Tosoh Corporation, Tokyo, Japan). All of the measurements were performed by SRL Inc. (Tokyo, Japan)

\section{Study outcome}

The primary outcome measure was plasma glucose levels during an OGTT. Secondary outcome measures included serum insulin, plasma glucagon levels, and endocrine hormone levels (cortisol, GH, noradrenaline and adrenaline levels).

The sample size of the current pilot study was determined with reference to previous clinical trials of conventional exercise, which demonstrated a significant acute phase effect of conventional exercise on post-load plasma glucose levels, with the sample size between 6 and 18 participants [10-14].

\section{Statistical analysis}

We primarily treated data without parametric assumptions and therefore reported the data as medians interquartile ranges (IQRs). However, parametric statistics (means and standard deviations [SDs]) would be sometimes more informative and therefore the values were additionally reported. Glucose, insulin, and glucagon levels at each time point in the WBVE $\rightarrow$ OGTT and OGTT $\rightarrow$ WBVE interventions were compared to the corresponding levels in the OGTT-alone intervention, using the Wilcoxon signed rank 
test. The effect size $r$ for the Wilcoxon signed rank test $(z$ score divided by the square root of $n$ ), and Cohen's $d$ (the standardized difference calculated from means and SDs) were also demonstrated. Effect size $r$ was categorized as small (0.1), medium (0.3), or large $(0.5)$, and $d$ was categorized as small (0.2), medium (0.5), or large (0.8) [19]. As the sensitivity analysis, the between-intervention differences were tested using the linear mixed model in which random effects for participants, fixed effects for the period, the allocated sequence, and the intervention were included. We also compared the change (delta) from baseline ( 0 min) between the interventions, using the Wilcoxon signed rank test.

Cortisol, GH, noradrenalin and adrenaline levels after WBVE were compared to those at the corresponding time point in the control intervention, using the Wilcoxon signed rank test. The association between the WBVE-to-OGTT interval and study outcomes in the WBVE $\rightarrow$ OGTT intervention were investigated using the Spearman's rank correlation method.

All statistical analyses were performed using $\mathrm{R}$ version 4.0.3 (R Development Core Team, Vienna, Austria).

\section{Results}

All of the 18 study participants completed the study, and were included in the analysis. The population had median and mean age of 33 (31-36) and $34 \pm 5$ years, and median and mean body mass index levels of 21 (19-23) and $22 \pm$ $5 \mathrm{~kg} / \mathrm{m}^{2}$. The median and mean WBVE-to-OGTT interval was $35(20-50)$ and $35 \pm 18 \mathrm{~min}$ in the WBVE $\rightarrow$ OGTT intervention.

Figure 2A shows plasma glucose levels during an OGTT. Plasma glucose levels in the WBVE $\rightarrow$ OGTT and OGTT $\rightarrow$ WBVE interventions were not significantly different from those in the OGTT-alone intervention at any time point except $15 \mathrm{~min}$, wherein plasma glucose levels were significantly higher in the WBVE $\rightarrow$ OGTT intervention (111 [102-122], $111 \pm 14 \mathrm{mg} / \mathrm{dL}$ vs 122 [111-134], $124 \pm 19 \mathrm{mg} / \mathrm{dL}, P=0.026$ by the Wilcoxon signed rank test). Serum insulin levels in the WBVE $\rightarrow$ OGTT intervention were significantly higher than those in the OGTTalone intervention at $0 \mathrm{~min}(4.7$ [3.7-5.7], $5.3 \pm 3.3 \mu \mathrm{U} / \mathrm{mL}$ vs 3.7 [2.6-4.9], $4.1 \pm 2.2 \mu \mathrm{U} / \mathrm{mL}, P=0.008$ by the Wilcoxon signed rank test), but not at any other time point (all $P>0.05$ by the Wilcoxon signed rank test) (Fig. 2B). Higher plasma glucagon levels were observed at $0 \mathrm{~min}$ in the WBVE $\rightarrow$ OGTT intervention and at $60 \mathrm{~min}$ in the OGTT $\rightarrow$ WBVE intervention (14.7 [11.8-20.3], 16.2 \pm $6.0 \mathrm{pg} / \mathrm{mL}$ vs $19.3[15.3-27.0], 23.7 \pm 16.3 \mathrm{pg} / \mathrm{mL}, P=$ 0.010 by the Wilcoxon signed rank test, and 4.2 [1.8-7.5], $6.1 \pm 5.1 \mathrm{pg} / \mathrm{mL} \quad$ vs 6.9 [5.1-10.6], $10.4 \pm 11.5 \mathrm{pg} / \mathrm{mL}$,

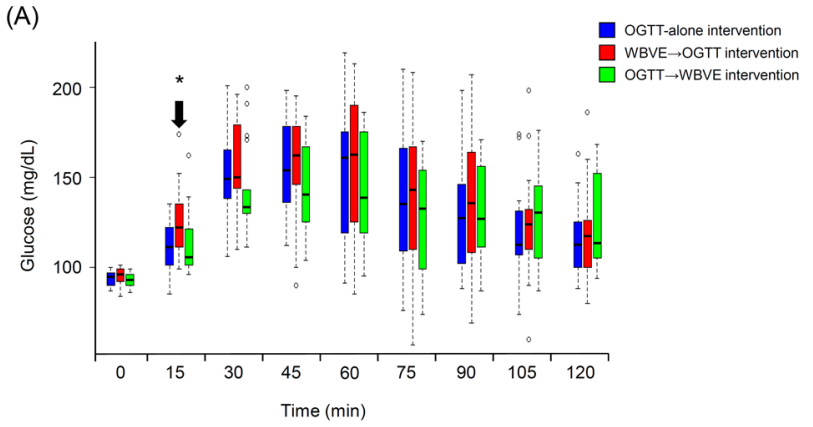

(B)
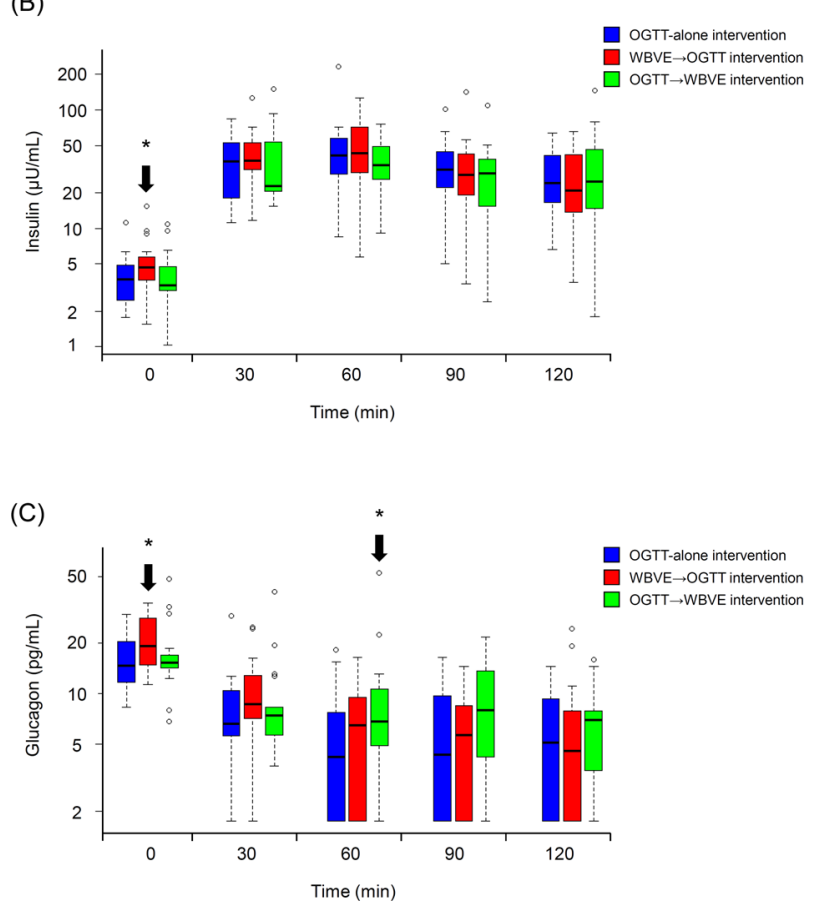

Fig. 2 Glucose (A), insulin (B), and glucagon levels (C) during an OGTT. Boxes represent interquartile ranges, whereas horizontal lines in respective boxes indicate medians. Whiskers represent ranges (minimum and maximum values) except outliers, which fall $>1.5$ times the interquartile range above the third quartile or below the first quartile. Axes of insulin (B) and glucagon (C) levels were a logarithmic scale. Arrows with an asterisk indicate $P<0.05$ vs the control intervention by the Wilcoxon signed rank test

$P=0.015$ by the Wilcoxon signed rank test, respectively) (Fig. 2C). The sensitivity analysis using the linear mixed model showed similar results (Supplementary Table 1). The effect sizes in the sample are demonstrated in Supplementary Table 2 . All measurements with statistically significant difference consistently had an effect size $r$ of larger than 0.5 , categorized as "large". On the other hand, those without significant difference consistently had a smaller $r$, categorized as "medium" or smaller; none of them were categorized as "large". The effect size $d$ was larger than 0.5 , categorized as "medium" or larger, in measurements with significant difference, and smaller in those without it; the 
Table 1 Endocrine hormone levels 10 min after WBVE

\begin{tabular}{|c|c|c|c|}
\hline & OGTT-alone intervention & WBVE $\rightarrow$ OGTT intervention & OGTT $\rightarrow$ WBVE intervention \\
\hline \multicolumn{4}{|c|}{ Before OGTT: 10 min after WBVE in the WBVE $\rightarrow$ OGTT intervention and the corresponding time point in the control intervention } \\
\hline $\begin{array}{l}\text { Growth hormone } \\
(\mathrm{ng} / \mathrm{mL})\end{array}$ & $0.26[0.14,0.98], 1.05 \pm 1.90$ & $3.26[2.72,6.92], 5.25 \pm 5.70,(P<0.001)$ & N/A \\
\hline Cortisol $(\mu \mathrm{g} / \mathrm{dL})$ & $7.0[5.5,8.8], 6.98 \pm 2.07$ & $8.5[7.2,13.5], 10.6 \pm 5.5,(P=0.026)$ & N/A \\
\hline $\begin{array}{l}\text { Noradrenaline } \\
(\mathrm{pg} / \mathrm{mL})\end{array}$ & $269[201,369], 310 \pm 147$ & $336[303,406], 351 \pm 75,(P=0.167)$ & N/A \\
\hline Adrenaline $(\mathrm{pg} / \mathrm{mL})$ & $29[24,32], 32 \pm 13$ & $55[42,77], 59 \pm 25,(P<0.001)$ & N/A \\
\hline \multicolumn{4}{|c|}{ During OGTT: $10 \mathrm{~min}$ after WBVE in the OGTT $\rightarrow$ WBVE intervention and the corresponding time point in the control intervention } \\
\hline $\begin{array}{l}\text { Growth hormone } \\
(\mathrm{ng} / \mathrm{mL})\end{array}$ & $0.11[0.04,0.20], 0.15 \pm 0.16$ & N/A & $0.28[0.11,0.68], 0.53 \pm 0.66,(P=0.006)$ \\
\hline Cortisol ( $\mu \mathrm{g} / \mathrm{dL})$ & $7.4[4.6,9.2], 6.9 \pm 2.6$ & N/A & $8.8[6.0,12.7], 10.1 \pm 5.4,(P=0.024)$ \\
\hline $\begin{array}{l}\text { Noradrenaline } \\
(\mathrm{pg} / \mathrm{mL})\end{array}$ & $344[248,441], 363 \pm 156$ & N/A & $306[245,446], 341 \pm 120,(P=0.551)$ \\
\hline Adrenaline (pg/mL) & $23[18,33], 25 \pm 11$ & N/A & $40[24,50], 42 \pm 22,(P<0.001)$ \\
\hline
\end{tabular}

Date are medians [interquartile ranges], means \pm standard deviations, as well as $P$ values versus the OGTT-alone intervention, tested by the Wilcoxon signed rank test

N/A not assessed

exception was 60-minute glucagon levels in the OGTT $\rightarrow$ WBVE versus OGTT-alone intervention, which reached statistical significance $(P<0.05$ by the Wilcoxon signed rank test) but had a "small" effect size $d$ (equal to 0.384). The comparison of deltas is shown in Supplementary Table 3.

Cortisol, GH, and adrenaline levels after WBVE were significantly higher than those at the corresponding time points in the OGTT-alone interventions, whereas noradrenaline levels were not. (Table 1).

In the WBVE $\rightarrow$ OGTT intervention, the interval between WBVE and OGTT had no significant correlation with any study outcomes (Supplementary Table 4).

There were no adverse events during the trial.

\section{Discussion}

The current pilot study demonstrated that plasma glucose levels in the WBVE $\rightarrow$ OGTT and OGTT $\rightarrow$ WBVE interventions were not significantly different from those in the OGTT-alone intervention at any time point except $15 \mathrm{~min}$, wherein plasma glucose levels were significantly higher in the WBVE $\rightarrow$ OGTT intervention. In the WBVE $\rightarrow$ OGTT intervention, serum insulin levels at 0 min were significantly higher, and plasma glucagon levels at $0 \mathrm{~min}$ were significantly higher compared to the OGTT-alone intervention. In the OGTT $\rightarrow$ WBVE intervention, post-load plasma glucagon levels were significantly higher at $60 \mathrm{~min}$ than in the OGTT-alone intervention, whereas serum insulin levels were not significantly different at any point compared to the OGTT-alone intervention. Plasma cortisol, GH and adrenaline levels at $10 \mathrm{~min}$ after WBVE were significantly higher than those at the corresponding time points in the OGTT-alone interventions; on the other hand, noradrenaline levels were not significantly changed with WBVE. The effect size in the sample $r$ was categorized consistently as "large" in the measurements with significant difference and "medium" or smaller in those without significant difference. Furthermore, the effect size in the sample $d$ was categorized as "medium" or larger in those with significant difference and as smaller in those without significant difference; the exception was 60-minute glucagon levels in the OGTT $\rightarrow$ WBVE vs OGTT-alone intervention, which reached statistical significance but had a "small" effect size $d$.

The additional analysis on the change (delta) from baseline showed that delta plasma glucose levels in the WBVE $\rightarrow$ OGTT and OGTT $\rightarrow$ WBVE interventions were not significantly different from those in the OGTT-alone intervention at any time point except $15 \mathrm{~min}$, wherein plasma glucose levels were significantly higher in the WBVE $\rightarrow$ OGTT intervention. These results were similar to the primary analysis. Delta serum insulin levels in the WBVE $\rightarrow$ OGTT and OGTT $\rightarrow$ WBVE interventions were not significantly different from those in the OGTT-alone intervention at any time point. Higher delta plasma glucagon levels were observed at $60 \mathrm{~min}$ in the OGTT $\rightarrow$ WBVE intervention, which was similar to the primary analysis. In contrast, lower delta plasma glucagon levels were observed at 60,90 , and $120 \mathrm{~min}$ in the WBVE $\rightarrow$ OGTT intervention, which would be caused by the elevation of absolute plasma glucagon levels at $0 \mathrm{~min}$ in the WBVE $\rightarrow$ OGTT intervention.

Previous studies demonstrated that conventional moderate-intensity exercise both before and after glucose 
ingestion decreased post-load glucose levels at the acute phase [10-14]. By contrast, WBVE did not decrease postload glucose levels either before or after glucose ingestion; rather, post-load plasma glucose levels were increased at $15 \mathrm{~min}$ in the WBVE $\rightarrow$ OGTT intervention. WBVE might have a different acute phase effect on post-load glucose metabolisms from conventional exercise.

The difference might be at least partially explained by altered endocrinological responses. After WBVE, GH levels were elevated compared with rest, and this response was apparently consistent with that observed after conventional exercise [20]. However, a recent study demonstrated that GH levels were higher after WBVE (i.e., exercise using WBV) than after exercise without WBV, suggesting that the stimulation of WBV per se would additionally increase GH levels [21]. Glucagon and cortisol also appeared to be secreted somewhat differently. Previous studies reported that conventional moderate-intensity exercise did not significantly affect glucagon or cortisol levels [22, 23]. By contrast, in the WBVE $\rightarrow$ OGTT intervention, glucagon levels at 0 min were significantly higher than those in the OGTT-alone intervention, and similar trend was seen at $30 \mathrm{~min}$ although the difference was not significant. Similarly, in the OGTT $\rightarrow$ WBVE intervention, glucagon levels at $60 \mathrm{~min}$ were significantly higher than the OGTT-alone intervention, although the parametric effect size in the sample $d$ was "small", suggesting that from the parametric viewpoint, its difference in the sample might be smaller than that of the other measurements with statistical significance. Furthermore, cortisol levels after WBVE in the WBVE $\rightarrow$ OGTT and OGTT $\rightarrow$ WBVE interventions were significantly higher than those at the corresponding time points in the OGTT-alone intervention. The increase of these counterregulatory hormones after WBVE might contribute to a non-decrease or even increase of postload glucose levels.

Cardinale $\mathrm{M}$ and colleagues demonstrated that cortisol levels were higher after WBVE (i.e., exercise using WBV) than after exercise without WBV, suggesting that the stimulation of WBV would increase cortisol levels [24]. The increase of cortisol levels after WBVE compared with rest in the current study would reflect the body response to the WBV stimulation. Several studies failed to detect a significant elevation of cortisol levels after WBVE $[21,25,26]$, which might come from their small and insufficient sample size $(n=6-8)$. Other studies reported that decreased cortisol levels after WBVE [27, 28]. However, those studies compared cortisol levels after WBVE to those before the WBVE, and not to those in the control at the corresponding time point. The decrease of cortisol in their studies might reflect the circadian rhythm of cortisol, which has a peak around wake-up time and a subsequent drop [29]. The current study, comparing cortisol levels after WBVE with those at rest at the same time point, would successfully detect the increase of cortisol levels by the intervention.

Catecholamine levels are another important factor increasing plasma glucose levels. Previous studies demonstrated that noradrenaline rather than adrenaline levels were predominantly increased after conventional exercise [20]. Conversely, in the current study, adrenaline levels were significantly elevated 10 min after WBVE, whereas noradrenaline levels were not. Previous studies reported that adrenaline had a stronger effect on the increase of plasma glucose levels than noradrenaline [30]. The adrenaline-dominant elevation might be also associated with altered post-load glucose metabolisms after WBVE. Loreto CD et al. reported a significant elevation of noradrenaline levels $35 \mathrm{~min}$ after the end of WBVE [31]. Although we did not measure catecholamine levels at the same time point, such late alternations of catecholamine levels might also contribute to a non-decrease or even increase of post-load glucose levels. The current study observed a temporal increase of insulin levels after WBVE, which would reflect an increased insulin requirement against those elevated counterregulatory hormones levels. This was in contrast to conventional exercise, where post-load insulin levels were decreased $[10,11,13,14]$, indicating decreased insulin requirements.

Why GH, glucagon, cortisol, and adrenaline levels were excessively increased after WBVE remained unknown. However, these findings suggest the body's overreaction to the exercise, or a somewhat stronger response than expected from conventional exercise. During exercise, contracting skeletal muscle rapidly increases glucose uptake via glucose transporter type 4 (GLUT4) translocation to the plasma membrane $[32,33]$. The body can appropriately balance glucose supply with the sudden, arbitrary demand, and successfully avoid hypoglycemia, wherein the liver plays a major role [34]. The physiological mechanisms of the delicate systemic balance, however, has not been completely understood. Humans might have acquired the delicate balance partially in an empirical manner during the process of evolution. WBVE is a novel exercise modality that humans have never experienced before. The body's empirical endocrinological response might result in an overreaction relative to the muscle's glucose consumption. These endocrinological overreactions might induce excess hepatic glucose release, leading to an insufficient suppression of post-load glucose levels.

Another factor potentially relevant to the altered glucose metabolisms is the glucose demand of the skeletal muscle. Previous studies reported that glucose uptake of the skeletal muscle during isometric contraction was different from that during isotonic contraction accompanied by a larger change of the muscle length [35]. During WBV static squatting, the skeletal muscle kept isometric from a macro point of view, while it was exposed to three-dimensional vibration load from a micro point of view. Muscle contraction during WBVE might be different from that during conventional 
exercise, and the difference might affect the glucose demand of the skeletal muscle.

The current findings indicate that WBVE, expected to have long-term beneficial effects on glucose metabolism like conventional moderate-intensity exercise, would affect post-load glucose metabolisms in a different way from conventional moderate-intensity exercise at the acute phase. WBVE might not be an equivalent or alternative modality to conventional exercise; potential benefits and risks might be different from those expected from abundant evidence of conventional exercise. Many more physiological and clinical studies will be needed to accumulate the evidence of WBVE.

Our study has some limitations. First, the sample size was limited. The effect size in the sample $r$ was always large in the measurements with significant difference (and $d$ was medium or large, except for 60-min glucagon levels in the OGTT $\rightarrow$ WBVE intervention), whereas it was consistently smaller in those without significant difference. However, from the viewpoint of the statistical power, the current sample size $(n=18)$ could detect the population effect size (not the sample effect size) $r>0.720$ by the Wilcoxon signed rank test (and $d>0.701$ by the paired $t$ test) with a power of $80 \%$ (calculated by G*Power version 3.1.9.2, Heinrich-Heine-Universität Düsseldorf, Düsseldorf, Germany), meaning that a difference with a smaller effect size in the population might be overlooked. Future studies with a larger sample size will be needed to detect such differences with a smaller effect size. Second, the current study protocol did not include an intervention of conventional (i.e., WBV-free) exercise. We were therefore unable to compare WBVE with conventional exercise directly, and to distinguish the effect of the WBV stimulation from that of exercise per se. Third, the current study did not assess hepatic glucose production, muscle glucose uptake, and actual muscle contraction. Fourth, the subjects of the current study were healthy volunteers. It remained unknown whether the similar results would be obtained from patients with diabetes. Fifth, the effect of unmeasured endocrine hormones remained unclear. Some studies examined testosterone, insulin-like growth factor 1 levels after WBVE $[24,36,37]$, which might contribute to post-load glucose metabolism after WBVE. Future studies will be needed to validate the current findings.

In conclusions, WBVE either following or followed by glucose ingestion did not decrease post-load plasma glucose levels like conventional moderate-intensity exercise.

\section{Availability of data}

The datasets generated and analysed during the current study are not publicly available due to ethical reason, but available from the corresponding author on reasonable request and with permission of the Institutional Review Board of Osaka University Hospital.

Author contributions H.W. designed the study, wrote the manuscript and researched date. M.T. designed the study and wrote the manuscript. N.K. contributed to discussion and reviewed/edited the manuscript. T.K., K.N. and I.S. reviewed/edited the manuscript. M.T. is the guarantor of this work and, as such, had full access to all the data in the study and takes responsibility for the integrity of the data and the accuracy of the date analysis.

\section{Compliance with ethical standards}

Conflict of interest The authors declare no competing interests.

Ethics approval The study was in accordance with the Declaration of Helsinki, and approved by the Institutional Review Board of Osaka University Hospital (date of approval, May 10, 2019; approval number, 18452-3).

Consent to participate Informed consent was obtained from all individual participants included in the study.

Publisher's note Springer Nature remains neutral with regard to jurisdictional claims in published maps and institutional affiliations.

\section{References}

1. D. Aune, T. Norat, M. Leitzmann, S. Tonstad, L.J. Vatten, Physical activity and the risk of type 2 diabetes: a systematic review and dose-response meta-analysis. Eur. J. Epidemiol. 30, 529-542 (2015). https://doi.org/10.1007/s10654-015-0056-Z

2. D.A. Bennett, H. Du, F. Bragg, Y. Guo, N. Wright, L. Yang, Z. Bian, Y. Chen, C. Yu, S. Wang, F. Meng, J. Lv, J. Chen, L. Li, R. Clarke, Z. Chen; China Kadoorie Biobank Study Collaborative Group., Physical activity, sedentary leisure-time and risk of incident type 2 diabetes: a prospective study of 512000 Chinese adults. BMJ Open Diabetes Res. Care. 7, e000835 (2019). https:// doi.org/10.1136/bmjdrc-2019-000835

3. M.S. Yerramalla, A. Fayosse, A. Dugravot, A.G. Tabak, M. Kivimäki, A. Singh-Manoux, S. Sabia, Association of moderate and vigorous physical activity with incidence of type 2 diabetes and subsequent mortality: 27 year follow-up of the Whitehall II study. Diabetologia 63, 537-548 (2020). https://doi.org/10.1007/ s00125-019-05050-1

4. M. Cardinale, C. Bosco, The use of vibration as an exercise intervention. Exerc. Sport Sci. Rev. 31, 3-7 (2003). https://doi. org/10.1097/00003677-200301000-00002

5. D.J. Cochrane, Vibration exercise: the potential benefits. Int. J. Sports Med. 32, 75-99 (2011). https://doi.org/10.1055/s-00301268010

6. C. Milanese, F. Piscitelli, M.G. Zenti, P. Moghetti, M. Sandri, C. Zancanaro, Ten-week whole-body vibration training improves body composition and muscle strength in obese women. Int. J. Med. Sci. 10, 307-311 (2013). https://doi.org/10.7150/ijms.5161

7. A. Bogaerts, C. Delecluse, A.L. Claessens, W. Coudyzer, S. Boonen, S.M.P. Verschueren, Impact of whole-body vibration training versus fitness training on muscle strength and muscle mass in older men: a 1-year randomized controlled trial. J. Gerontol. A Biol. Sci. Med. Sci. 62, 630-635 (2007). https://doi.org/ 10.1093/gerona/62.6.630

8. A. Bellia, M. Salli, M. Lombardo, M. D’Adamo, V. Guglielmi, C. Tirabasso, C. Tirabasso, L. Giordani, M. Federici, D. Lauro, C. Foti, 
P. Sbraccia, Effects of whole body vibration plus diet on insulinresistance in middle-aged obese subjects. Int. J. Sports Med. 35, 511-516 (2014). https://doi.org/10.1055/s-0033-1354358

9. S.R. Colberg, R.J. Sigal, J.E. Yardley, M.C. Riddell, D.W. Dunstan, P.C. Dempsey, E.S. Horton, K. Castorino, D.F. Tate, Physical activity/exercise and diabetes: a position statement of the American diabetes association. Diabetes Care. 39, 2065-2079 (2016). https://doi.org/10.2337/dc16-1728

10. J.D. Nelson, P. Poussier, E.B. Marliss, A.M. Albisser, B. Zinman, Metabolic response of normal man and insulin-infused diabetics to postprandial exercise. Am. J. Physiol. 242, E309-316 (1982). https://doi.org/10.1152/ajpendo.1982.242.5.E309

11. J. Achten, A.E. Jeukendrup, Effects of pre-exercise ingestion of carbohydrate on glycaemic and insulinaemic responses during subsequent exercise at differing intensities. Eur. J. Appl Physiol. 88, 466-471 (2003). https://doi.org/10.1007/s00421-002-0730-1

12. A.T. Hostmark, G.S. Ekeland, A.C. Beckstrom, H.D. Meen, Postprandial light physical activity blunts the blood glucose increase. Prev. Med. 42, 369-371 (2006). https://doi.org/10.1016/ j.ypmed.2005.10.001

13. K.R. Short, L.V. Pratt, A.M. Teague, The acute and residual effect of a single exercise session on meal glucose tolerance in sedentary young adults. J. Nutr. Metab. 2012, 278678 (2012). https://doi. org/10.1155/2012/278678

14. Y.H. Shin, H.L. Jung, J.W. Ryu, P.S. Kim, T.Y. Ha, J.Y. An, H.Y. Kang, Effects of a pre-exercise meal on plasma growth hormone response and fat oxidation during walking. Prev. Nutr. Food Sci. 18, 175-180 (2013). https://doi.org/10.3746/pnf.2013.18.3.175

15. E. Chacko, Exercising tactically for taming postmeal glucose surges. Sci. (Cairo). 2016, 4045717 (2016). https://doi.org/10. $1155 / 2016 / 4045717$

16. J. Haxhi, P.A. Scotto di, M. Sacchetti, Exercising for metabolic control: is timing important? Ann. Nutr. Metab. 62, 14-25 (2013). https://doi.org/10.1159/000343788

17. N.J. Wewer Albrechtsen, B. Hartmann, S. Veedfald, J.A. Windelov, A. Plamboeck, K.N. Bojsen-Moller, T. Idorn, B. Feldt-Rasmussen, F. K. Knop, T. Vilsbøll, S. Madsbad, C.F. Deacon, J.J. Holst, Hyperglucagonaemia analysed by glucagon sandwich ELISA: nonspecific interference or truly elevated levels? Diabetologia 57, 1919-1926 (2014). https://doi.org/10.1007/s00125-014-3283-z

18. T. Matsuo, J. Miyagawa, Y. Kusunoki, M. Miuchi, T. Ikawa, T. Akagami, M. Tokuda, T. Katsuno, A. Kushida, T. Inagaki, M. Namba, Postabsorptive hyperglucagonemia in patients with type 2 diabetes mellitus analyzed with a novel enzyme-linked immunosorbent assay. J. Diabetes Investig. 7, 324-331 (2016). https://doi. org/10.1111/jdi.12400

19. J. Cohen. Statistical power analysis for the behavioral sciences. 2nd ed. Lawrence Erlbaum Associates, Hillside, NJ, (1988)

20. A. Weltman, C.J. Pritzlaff, L. Wideman, J.Y. Weltman, J.L. Blumer, R.D. Abbott, M.L. Hartman, J.D. Veldhuis, Exercisedependent growth hormone release is linked to markers of heightened central adrenergic outflow. J. Appl. Physiol. (1985) 89 629-635 (2000). https://doi.org/10.1152/jappl.2000.89.2.629

21. A.E. Rigamonti, A. De Col, S. Tamini, G. Tringali, R. De Micheli, L. Abbruzzese, Goncalves, C.R. da Cruz, M. Bernardo-Filho, S.G. Cella, A. Sartorio, GH responses to whole body vibration alone or in combination with maximal voluntary contractions in obese male adolescents. Growth Horm. IGF Res. 42-43, 22-27 (2018). https://doi.org/10.1016/j.ghir.2018.07.004

22. A. Luger, P.A. Deuster, S.B. Kyle, W.T. Gallucci, L.C. Montgomery, P.W. Gold, D.L. Loriaux, G.P. Chrousos, Acute hypothalamic-pituitary-adrenal responses to the stress of treadmill exercise. Physiologic Adapt. Phys. Train. N. Engl. J. Med. 316, 1309-1315 (1987). https://doi.org/10.1056/NEJM198705213162105
23. E.B. Marliss, M. Vranic, Intense exercise has unique effects on both insulin release and its roles in glucoregulation: implications for diabetes. Diabetes 51, S271-283 (2002). https://doi.org/10. 2337/diabetes.51.2007.s271

24. M. Cardinale, R.L. Soiza, J.B. Leiper, A. Gibson, W.R. Primrose, Hormonal responses to a single session of wholebody vibration exercise in older individuals. Br. J. Sports Med. 44, 284-288 (2010). https://doi.org/10.1136/bjsm.2007.043232

25. M. Giunta, A.E. Rigamonti, F. Agosti, A. Patrizi, E. Compri, M. Cardinale, A. Sartorio, Combination of external load and whole body vibration potentiates the GH-releasing effect of squatting in healthy females. Horm. Metab. Res. 45, 611-616 (2013). https:// doi.org/10.1055/s-0033-1341464

26. J. Erskine, I. Smillie, J. Leiper, D. Ball, M. Cardinale, Neuromuscular and hormonal responses to a single session of whole body vibration exercise in healthy young men. Clin. Physiol. Funct. Imaging 27, 242-248 (2007). https://doi.org/10.1111/j. 1475-097X.2007.00745.X

27. M. Elmantaser, M. McMillan, K. Smith, S. Khanna, D. Chantler, M. Panarelli, S.F. Ahmed, A comparison of the effect of two types of vibration exercise on the endocrine and musculoskeletal system. J. Musculoskelet. Neuronal Interact. 12, 144-154 (2012)

28. C. Bosco, M. Iacovelli, O. Tsarpela, M. Cardinale, M. Bonifazi, J. Tihanyi, M. Viru, A. De Lorenzo, A. Viru, Hormonal responses to whole-body vibration in men. Eur. J. Appl Physiol. 81, 449-454 (2000). https://doi.org/10.1007/s004210050067

29. R.C. Bhake, V. Kluckner, H. Stassen, G.M. Russell, J. Leendertz, K. Stevens, A.C.E. Linthorst, S.L. Lightman, Continuous free cortisol profiles-circadian rhythms in healthy men. J. Clin. Endocrinol. Metab. 104, 5935-5947 (2019). https://doi.org/10. 1210/jc.2019-00449

30. H. Ensinger, T. Weichel, K.H. Lindner, A. Grünert, M. Georgieff, Are the effects of noradrenaline, adrenaline and dopamine infusions on VO2 and metabolism transient? Intensive Care Med. 21, 50-56 (1995). https://doi.org/10.1007/BF02425154

31. C. Di Loreto, A. Ranchelli, P. Lucidi, G. Murdolo, N. Parlanti, A. De Cicco, O. Tsarpela, G. Annino, C. Bosco, F. Santeusanio, G.B. Bolli, P. De Feo, Effects of whole-body vibration exercise on the endocrine system of healthy men. J. Endocrinol. Invest. 27, 323-327 (2004). https://doi.org/10.1007/BF03351056

32. T. Hayashi, J.F. Wojtaszewski, L.J. Goodyear, Exercise regulation of glucose transport in skeletal muscle. Am. J. Physiol. 273, E1039-1051 (1997). https://doi.org/10.1152/ajpendo.1997.273.6. E1039

33. E.A. Richter, M. Hargreaves, Exercise, GLUT4, and skeletal muscle glucose uptake. Physiol. Rev. 93, 993-1017 (2013). https://doi.org/10.1152/physrev.00038.2012

34. D.H. Wasserman, Four grams of glucose. Am. J. Physiol. Endocrinol. Metab. 296, E11-21 (2009). https://doi.org/10.1152/a jpendo. 90563.2008

35. O.E. Andersen, O.B. Nielsen, K. Overgaard, Early effects of eccentric contractions on muscle glucose uptake. J. Appl Physiol. (1985) 126, 376-385 (2019). https://doi.org/10.1152/japplphysiol. 00388.2018

36. M. Cardinale, J. Leiper, J. Erskine, M. Milroy, S. Bell, The acute effects of different whole body vibration amplitudes on the endocrine system of young healthy men: a preliminary study. Clin. Physiol. Funct. Imaging 26, 380-384 (2006). https://doi.org/ 10.1111/j.1475-097X.2006.00706.X

37. R. Di Giminiani, N. Rucci, L. Capuano, M. Ponzetti, F. Aielli, J. Tihanyi, Individualized whole-body vibration: neuromuscular, biochemical, muscle damage and inflammatory acute responses. Dose Response 18, 1559325820931262 (2020). https://doi.org/10. $1177 / 1559325820931262$ 